\title{
ON THE NODAL LINE OF THE SECOND EIGENFUNCTION OF THE LAPLACIAN IN $\mathbb{R}^{2}$
}

\author{
ANTONIOS D. MELAS
}

\section{Introduction}

A conjecture of L. Payne [8] states that any second eigenfunction of the Laplacian with zero boundary condition for a bounded domain $\Omega \subseteq \mathbb{R}^{2}$ does not have a closed nodal line. This is also asked by S.-T. Yau [10, Problem 78] for $\Omega$ a bounded convex domain in $\mathbb{R}^{2}$.

L. Payne [9] proved the conjecture provided the domain $\Omega \subseteq \mathbb{R}^{2}$ is symmetric with respect to one line and convex with respect to the direction vertical to this line. Also. C.-S. Lin [7] proved the conjecture provided the domain $\Omega \subseteq \mathbb{R}^{2}$ is smooth, convex, and invariant under a rotation with angle $2 \pi p / q$, where $p$ and $q$ are positive integers. Recently D. Jerison [5] proved the conjecture for long thin convex sets. Without any assumption on the smoothness of $\Omega$ he showed that the nodal line has to intersect $\partial \Omega$ in exactly two points.

In this paper we prove the conjecture when $\Omega$ is a bounded convex domain in $\mathbb{R}^{2}$ with $C^{\infty}$ boundary.

To fix the notation for a bounded domain $\Omega \subseteq \mathbb{R}^{2}$ with smooth boundary we let $u_{2}$ be a second eigenfunction of $\Omega$, that is, $u_{2}$ is a solution of the Dirichlet problem

$$
\begin{cases}\Delta u_{2}+\lambda_{2} u_{2}=0 & \text { in } \Omega, \\ u_{2}=0 & \text { on } \partial \Omega,\end{cases}
$$

where $\Delta=\sum_{i=1}^{2}\left(\partial^{2} / \partial x_{i}^{2}\right)$ and $\lambda_{2}$ is the second eigenvalue of $\Omega$.

The nodal line $N$ of $u_{2}$ is defined by

$$
N=\overline{\left\{x \in \Omega: u_{2}(x)=0\right\}} \text {. }
$$

The Courant nodal domain theorem implies that $N$ must divide the domain $\Omega$ into exactly two components.

Our main result is the following:

Received April 15, 1991. 
Theorem 1.1. If $\Omega \subseteq \mathbb{R}^{2}$ is a bounded convex domain with $C^{\infty}$ boundary, then the nodal line $N$ of any second eigenfunction $u_{2}$ must intersect the boundary $\partial \Omega$ at exactly two points.

Theorem 1.1 implies

Corollary 1.2. If $\Omega \subseteq \mathbb{R}^{2}$ is a bounded convex domain, then the nodal line $N$ of any second eigenfunction $u_{2}$ does not enclose a compact subregion of $\Omega$.

Proof. Otherwise we can approximate $\Omega$ by a convex domain $\widetilde{\Omega}$ with $C^{\infty}$ boundary for which the nodal line of a second eigenfunction of $\widetilde{\Omega}$ still encloses a compact subregion of $\Omega$ and this contradicts Theorem 1.1.

In $\S 2$ we show as in [7] that if Theorem 1.1 is false, then we can find a bounded convex domain $\Omega$ with $C^{\infty}$ boundary such that the nodal line $N$ of a second eigenfunction $u_{2}$ intersects $\partial \Omega$ at exactly one point $o$.

In $\S 3$ we prove that the above situation is impossible and this proves Theorem 1.1. To do that we introduce the functions $v_{t}=\partial u_{2} / \partial x_{1}+t u_{2}$ for $t \in \mathbb{R}$ where the $x_{1}$-direction is tangent to $\partial \Omega$ at $o$.

We show that for each $t \in \mathbb{R}$ there exists exactly one subdomain $\Omega_{t}$ of $\Omega$ such that $v_{t}=0$ on $\partial \Omega_{t}$. Since $\Delta v_{t}+\lambda_{2} v_{t}=0$ in $\Omega$, it is concluded that either $v_{t}$ or $-v_{t}$ is everywhere positive in $\Omega_{t}$. Then defining $A=\left\{t \in \mathbb{R}: v_{t}>0\right.$ in $\left.\Omega_{t}\right\}$ and $B=\left\{t \in \mathbb{R}: v_{t}<0\right.$ in $\left.\Omega_{t}\right\}$ we prove that $A, B$ are closed subsets of $\mathbb{R}$ and finally that both are nonempty. This gives a contradiction since $\mathbb{R}=A \cup B$ and $A \cap B=\varnothing$.

Also we need a technical lemma which allows us to control the singularity of $N$ on $\partial \Omega$. It is because of this that we have to assume $\Omega$ has $C^{\infty}$ boundary. We will state this lemma in $\S 2$ but we prove it in $\S 4$.

2.

Let $(C)$ be the proposition: "The nodal line of any second eigenfunction of $\Omega$ intersects $\partial \Omega$ at exactly two points." Then Theorem 1.1 means that $(C)$ is true for any bounded convex domain $\Omega$ with $C^{\infty}$ boundary. For such domains we have the following technical lemma.

Lemma 2.1. Let $\Omega \subseteq \mathbb{R}^{2}$ be a bounded convex domain with $C^{\infty}$ boundary, let $q \in \partial \Omega$, and $r_{0}>0$, and suppose $u$ satisfies

$$
\begin{cases}\Delta u+\lambda u=0 & \text { in } \Omega, \\ u=0 & \text { in }(\partial \Omega) \cap D\left(q ; r_{0}\right),\end{cases}
$$

where $\lambda$ is a constant, and $D\left(q, r_{0}\right)$ denotes the disc of radius $r_{0}$ centered 
at $q$. Also assume that the $x_{1}$-axis is in the direction of the tangent of $\partial \Omega$ at $q$. Then we have the following.

(i) $u$ does not vanish of infinite order at $q$.

(ii) If the nodal line of $u$ is $N$, and $q \in N$, then $N$ approaches $q$ nontangentially with respect to $\partial \Omega$.

(iii) There exists an $\varepsilon>0$ such that

$$
|u|+\left|\partial u / \partial x_{1}\right|>0 \quad \text { in } \Omega \cap\{x: 0<|x-q|<\varepsilon\}
$$

This lemma will be proved in $\S 4$.

The following lemmas are from [7].

Lemma 2.2. Suppose $p \in \partial \Omega$ and that $\Omega$ has $C^{2}$ boundary. Then $\left(\partial u_{2} / \partial \nu\right)(0)=0$ if and only if $p \in N$, where $\partial u_{2} / \partial \nu$ is the outward normal derivative of $u_{2}$ on $\partial \Omega$.

Lemma 2.3. Let $u_{2}$ be a second eigenfunction of $\Omega$. If $\partial u_{2} / \partial \nu \geq 0$ on $\partial \Omega$, then up to multiplication by a constant, $u_{2}$ is the only second eigenfunction of $\Omega$.

Theorem 2.1. Suppose $\Omega_{0}$ is a bounded convex domain with $C^{\infty}$ boundary such that $(C)$ fails for $\Omega_{0}$. Then there exists a convex bounded domain $\Omega$ with $C^{\infty}$ boundary and a second eigenfunction $u_{2}$ of $\Omega$ such that $\partial u_{2} / \partial \nu$ has exactly one zero on $\partial \Omega$.

Proof. This was proved by C.-S. Lin [7]. We sketch the proof here for completeness. Let $\Omega(t)$ be a smooth deformation with $\Omega(0)=\Omega_{0}$ and $\Omega(1)$ a disc such that $\Omega(t)$ is a bounded convex domain with $C^{\infty}$ boundary. Since $(C)$ fails for $\Omega_{0}$ and obviously holds for $\Omega(1)$, we may define $t_{0}=\sup \{t \in[0,1]:(C)$ fails for $\Omega(t)\}$ and we have $0 \leq$ $t_{0}<1$. Thus there exist sequences $t_{i}, \tilde{t}_{i}$ with $t_{i} \leq t_{0} \leq \tilde{t}_{i}$ and $t_{i}$, $\tilde{t}_{i} \rightarrow t_{0}$ as $i \rightarrow+\infty$ and such that there exist normalized eigenfunctions $u_{i}, \tilde{u}_{i}$ of $\Omega\left(t_{i}\right), \Omega\left(\tilde{t}_{i}\right)$ respectively such that $\partial u_{i} / \partial \nu \geq 0$ on $\partial \Omega\left(t_{i}\right)$ and $\partial \Omega \tilde{u}_{i} / \partial_{\nu}$ has at least two zeros on $\partial \Omega\left(\tilde{t}_{i}\right)$. Since the second eigenvalue of $\Omega(t)$ is a continuous function of $t$, [3], we can by elliptic estimates get subsequences of $\left\{u_{i}\right\}$ and $\left\{\tilde{u}_{i}\right\}$ converging to $u_{0}, \tilde{u}_{0}$ so that $u_{0}, \tilde{u}_{0}$ are second eigenfunctions of $\Omega\left(t_{0}\right)$, and moreover $\partial u_{0} / \partial \nu \geq 0$ on $\partial \Omega\left(t_{0}\right)$, and $\partial \tilde{u}_{0} / \partial \nu$ has at least one zero on $\partial \Omega\left(t_{0}\right)$. By Lemma 2.3 we may assume $u_{0}=\tilde{u}_{0}$. Hence $\partial u_{0} / \partial \nu$ has at least on zero on $\partial \Omega\left(t_{0}\right)$ and does not change sign on $\partial \Omega\left(t_{0}\right)$. But from Lemmas 2.1 (ii) and 2.2 it follows that zeros of $\partial u_{0} / \partial \nu$ on $\partial \Omega\left(t_{0}\right)$ are isolated. Since the nodal line of $u_{0}$ divides $\Omega\left(t_{0}\right)$ into exactly two components and $u_{0}$ has opposite signs on each of them, the set of zeros of $\partial u_{0} / \partial \nu$ on $\partial \Omega\left(t_{0}\right)$ is connected. Hence 
$\partial u_{0} / \partial \nu$ has exactly one zero on $\partial \Omega\left(t_{0}\right)$. This proves the theorem with $\Omega=\Omega_{0}\left(t_{0}\right)$ and $u_{2}=u_{0}$.

\section{Proof of Theorem 1.1}

By Theorem 2.1 we conclude that Theorem 1.1 follows from:

Theorem 3.1. If $\Omega \subseteq \mathbb{R}^{2}$ is a bounded convex domain with $C^{\infty}$ boundary, and $u_{2}$ is a second eigenfunction of $\Omega$, then $\partial u_{2} / \partial \nu$ cannot have exactly one zero on $\partial \Omega$.

Proof. Assume $\partial u_{2} / \partial \nu$ vanishes at exactly one point $o \in \partial \Omega$. We choose the coordinate axes so that $o$ is the origin, the $x_{1}$-axis is tangent to $\partial \Omega$ at $o$, and the $x_{2}$-axis is in the direction of the inward normal to $\partial \Omega$ at $o$. Let $\Omega^{+}=\left\{x \in \Omega: u_{2}(x)>0\right\}$ and $\Omega^{-}=\left\{x \in \Omega: u_{2}(x)<0\right\}$. By Lemma 2.2, $N \cap \partial \Omega=\{o\}$. Also since by the maximum principle $u_{2}$ has to change sign near any point in $N \cap \Omega$, we may assume that $\partial \Omega^{-}=N$, and it is easy to see that $\Omega^{-}$is simply connected.

Since $\Omega$ is convex, the set of points $p$ on $\partial \Omega$, where the tangent to $\partial \Omega$ at $p$ is parallel to the $x_{1}$-axis, consists exactly of two closed line segments $I, J$ (which may be points) parallel to the $x_{1}$-axis. Assume $o \in I$ and let $\Gamma^{+}$and $\Gamma^{-}$be the two open subarcs of $\partial \Omega$, which form $\partial \Omega \backslash(I \cup J)$. Since $u_{2}$ is nonnegative near $\partial \Omega \backslash(I \cup J)$ by Hopf's boundary point lemma we may assume that

$$
\partial u_{2} / \partial x_{1}>0 \quad \text { in } \Gamma^{+} \quad \text { and } \quad \partial u_{2} / \partial x_{1}<0 \quad \text { in } \Gamma^{-} .
$$

Also it is obvious that

$$
\partial u_{2} / \partial x_{1}=0 \quad \text { on } I \cup J
$$

For any $t \in \mathbb{R}$ we define $v_{t}$ on $\bar{\Omega}$ by

$$
v_{t}(x)=e^{-t x_{1}} \frac{\partial}{\partial x_{1}}\left(e^{t x_{1}} u_{2}(x)\right)=\frac{\partial u_{2}}{\partial x_{1}}(x)+t u_{2}(x) .
$$

Then we have

$$
\Delta v_{t}+\lambda_{2} v_{t}=0 \quad \text { in } \quad \Omega,
$$

where $\lambda_{2}$ is the second eigenvalue of $\Omega$.

Let $N_{t}=\overline{\left\{x \in \Omega: v_{t}(x)=0\right\}}$ be the nodal line of $v_{t}$. By (3.1) we have $N_{t} \cap \partial \Omega \subseteq I \cup J$. Also $v_{t}=0$ on $I \cup J$. The basic lemma about $N_{t}$ is the following:

Lemma 3.1. For every $t \in \mathbb{R}$ there exists at least one subdomain $\Omega_{t}$ of $\Omega$ such that $\partial \Omega_{t} \subseteq N_{t} \cup I \cup J$. 
Proof. Clearly the gradient of $u_{2}$ vanishes at every point of $N \cap \Omega$, where $N$ is not $C^{1}$. By Lemma 2.1(ii), (iii) (for $u=u_{2}, q=0$ ) $N$ approaches $o$ nontangentially with respect to $\partial \Omega$, and there exists $\varepsilon>0$ such that $\partial u_{2} / \partial x_{1}$ has no zeros in $N \cap\{x: 0<|x|<\varepsilon\}$. Hence there exists $\eta>0$ such that if $0<\bar{\eta} \leq \eta$ and $\Sigma_{\bar{\eta}}=\left\{\left(x_{1}, x_{2}\right): 0<x_{2} \leq \bar{\eta}\right\}$, then $N \cap \Sigma_{\bar{\eta}}$ is a $C^{1}$ one-manifold with boundary on the line $x_{2}=\bar{\eta}$, and such that its tangent is never parallel to the $x_{1}$-axis. Hence $N \cap \Sigma_{\bar{\eta}}$ does not contain embedded circles or arcs with both endpoints on $x_{2}=\bar{\eta}$. Also $N \cap \partial \Omega=\{o\}$, and $N$ divides $\Omega$ into exactly two components. Thus $N \cap \Sigma_{\bar{\eta}}$ consists of two simple arcs each of which has one endpoint on $x_{2}=\bar{\eta}$ and approaches $o$. Hence $N \cap \Sigma_{\eta}$ consists of two simple arcs which approach $o$, and every line $x_{2}=\bar{\eta}$ for $0<\bar{\eta} \leq \eta$ intersects each of them exactly once. Let $\gamma_{1}$ be one of these arcs.

Now suppose there exists $t_{0} \in \mathbb{R}$ such that there exists no subdomain $\Omega^{\prime}$ of $\Omega$ with $\partial \Omega^{\prime} \subseteq N_{t_{0}} \cup I \cup J$. Then from (3.1), (3.2) and the fact that $\Gamma^{+}, \Gamma^{-}$are connected, it follows that $\bar{\Omega} \backslash\left(N_{t_{0}} \cup I \cup J\right)$ has exactly two components so that the sets $U^{+}=\left\{x \in \bar{\Omega}: v_{t_{0}}(x)>0\right\}$ and $U^{-}=\{x \in$ $\left.\bar{\Omega}: v_{t_{0}}(x)<0\right\}$ are both connected. We may assume that $\gamma_{1}$ lies in the interior of $U^{+}$. Since $\bar{\Gamma}_{1} \cap \partial U^{+}=\{o\}$ and $\gamma_{1}$ is a simple arc, $U^{+} \backslash \gamma_{1}$ is connected. So if $x=\left(x_{1}, \eta\right) \in \gamma_{1}$ and $y=\left(y_{1}, \eta\right) \in \Gamma^{+}$, we may join $x$ and $y$ by a simple polygonal path $\gamma_{2}$ such that $\gamma_{2} \backslash\{x, y\}$ lies in the interior of $U^{+} \backslash \gamma_{1}$. Let $\gamma_{3}$ be the subarc of $\Gamma^{+} \cup I$ with endpoints $o$ and $y$. Then $\gamma=\gamma_{1} \cup \gamma_{2} \cup \gamma_{3}$ is a simple closed curve, hence by the Jordan curve theorem there exists a unique bounded domain $W$ with $\partial W=\gamma$. Since $\Omega$ is simply connected, we have $W \subseteq \Omega$. Since $v_{t_{0}} \geq 0$ on $\gamma$, $\Gamma^{-} \cap \bar{W}=\varnothing$, and $U^{-}$is connected, we have $v_{t_{0}} \geq 0$ in $W$, so by the maximum principle $v_{t_{0}}>0$ in $W$.

Since $\gamma_{2}$ does not meet the line $x_{2}=0$, there exists $\eta_{1}$ with $0<\eta_{1} \leq \eta$ such that $\gamma_{2}$ does not meet the line $x_{2}=\eta_{1}$. This line meets $\gamma_{1}$ at exactly one point $\bar{x}$ and $\Gamma^{+}$at exactly one point $\bar{y}$. Hence the line segment $[\bar{x}, \bar{y}]$ intersects the curve $\gamma=\partial W$ at exactly $\bar{x}$ and $\bar{y}$. Since points on $[\bar{x}, \bar{y}]$ which are near $\Gamma^{+}$must be in $W$, we conclude that $[\bar{x}, \bar{y}]$ lies in $\bar{W}$. On the other hand, $e^{t_{0} x_{1}} u_{2}(x)$ vanishes on $\bar{x}$ and $\bar{y}$, hence by (3.3) there exists $z$ in the open segment with endpoints $\bar{x}$ and $\bar{y}$ such that $v_{t_{0}}(z)=0$. But then $z \in W$ and this is a contradiction since $v_{t_{0}}>0$ in $W$.

Lemma 3.2. For every $t \in \mathbb{R}$ we have the following: 
(i) There exists exactly one subdomain $\Omega_{t}$ of $\Omega$ such that $\partial \Omega_{t} \subseteq$ $N_{t} \cup I \cup J$.

(ii) Either $v_{t}>0$ in $\Omega_{t}$ or $v_{t}>0$ in $\Omega_{t}$.

(iii) $\lambda_{1}\left(\Omega_{t}\right)=\lambda_{2}$, where $\lambda_{1}\left(\Omega_{t}\right)$ is the first eigenvalue of $\Omega_{t}$ with respect to the Dirichlet boundary condition.

(iv) $\Omega_{t}$ is simply connected.

(v) There exists $\rho>0$ depending only on $\lambda_{2}$ such that $\Omega_{t}$ contains a disc of radius $\rho$.

Proof. Fix $t \in \mathbb{R}$. (i) Assume there exist two distinct subdomains of $\Omega$ whose boundary is contained in $N_{t} \cup I \cup J$. Then there are two disjoint subdomains $\Omega_{1}, \Omega_{2}$, of $\Omega$ such that $v_{t}=0$ on $\partial \Omega_{1} \cup \partial \Omega_{2}$. Since $\Delta v_{t}+\lambda_{2} v_{t}=0$ in $\Omega_{1} \cup \Omega_{2}$ and $\lambda_{2}$ is the second eigenvalue of $\Omega$, this yields a contradiction as in the proof of Courant's nodal domain theorem. Hence (i) follows from Lemma 3.1.

(ii) follows from (i).

(iii) follows from (i) and (ii) since $\Delta v_{t}+\lambda_{2} v_{t}=0$ in $\Omega_{t}$.

(iv) follows from (i) and the fact that $\Omega$ is simply connected.

(v) follows from (iii) and (iv) by using Hayman's inner radius theorem [4].

Lemma 3.3. There exist $C_{1}, C_{2}>0$ such that $v_{t}>0$ in $\Omega_{t}$ for every $t>C_{1}$ and $v_{t}>0$ in $\Omega_{t}$ for every $t<-C_{2}$.

Proof. Let $z \in \Gamma^{+}$. Since $\partial u_{2} / \partial x_{1}(z)>0$, there exists $\delta>0$ sufficiently small such that $\partial u_{2} / \partial x_{1}>0$ in $\bar{\Omega} \cap D(z ; \delta)$, and moreover since $z \in \partial \Omega^{+}$, we may assume that $D(z ; \delta) \cap \bar{\Omega}^{-}=\varnothing$. Let $K=\bar{\Omega} \cap D(z ; \delta)$. Then using $\lambda_{1}\left(\Omega^{+}\right)=\lambda_{2}$ and the monotonicity principle for eigenvalues, we have $\lambda_{1}\left(\Omega^{+} \backslash K\right)>\lambda_{2}$. Hence by the continuity of the first eigenvalue under continuous deformations of the domain [3] we can choose a sufficiently large compact subset $E$ of $\Omega^{-}$such that $\lambda_{1}(\Omega \backslash(K \cup E))>\lambda_{2}$. Let $M=\sup \left\{\left|\partial u_{2} / \partial x_{1}(x)\right|: x \in \Omega\right\}$ and $\alpha=\inf \left\{\left|u_{2}(x)\right|: x \in E\right\}>0$. Now fix any $t$ such that $t>\alpha^{-1} M$. Then

$$
v_{t}>0 \quad \text { in } K \quad \text { and } \quad v_{t}<0 \quad \text { in } E .
$$

Hence $N_{t} \cup I \cup J \subseteq \bar{\Omega} \backslash(K \cup E)$, and since $\bar{\Omega} \backslash K$ is simply connected we have $\Omega_{t} \subseteq \bar{\Omega} \backslash K$. Also from Lemma 3.2(iii) it follows that $\lambda_{1}\left(\Omega_{t}\right)=$ $\lambda_{2}<\lambda_{1}(\Omega \backslash(K \cup E))$ so that $\Omega_{t} \subseteq \Omega \backslash(K \cup E)$. Hence $\Omega_{t} \cap E \neq \varnothing$, and since $v_{t}<0$ in $E$ by Lemma 3.2(ii) we must have $v_{t}<0$ in $\Omega_{t}$ if $t>\alpha^{-1} M=C_{1}$. Similarly we can find $C_{2}>0$ such that $v_{t}<0$ in $\Omega_{t}$ if $t<-C_{2}$. 
Lemma 3.4. The sets $A=\left\{t \in \mathbb{R}: v_{t}>0\right.$ in $\left.\Omega_{t}\right\}$ and $B=\left\{t \in \mathbb{R}: v_{t}<\right.$ 0 in $\Omega_{t}$ \} are closed.

Proof. Assume $t_{j} \in A, t_{j} \rightarrow t_{0}$ as $j \rightarrow \infty$ but $t_{0} \notin A$. Then $v_{t_{j}}>0$ in $\Omega_{t_{j}}$ and by Lemma 3.2(ii) $v_{t_{0}}<0$ in $\Omega_{t_{0}}$. By Lemma 3.3(v) we can find discs $D\left(x_{j} ; \rho\right) \subseteq \Omega_{t_{j}}$. Taking a subsequence we may assume $x_{j} \rightarrow x_{0} \in \bar{\Omega}$. It follows then that $D\left(x_{0} ; \rho\right) \subseteq \bar{\Omega}$ and $v_{t_{0}} \geq 0$ in $D\left(x_{0} ; \rho\right)$. By the maximum principle, $v_{t_{0}}\left(x_{0}\right)>0$. Since $v_{t_{0}}<0$ in $\Omega_{t_{0}}$, Lemma 3.2(i) implies that $N_{t_{0}}$ does not separate $x_{0}$ from $\partial \Omega \backslash(I \cup J)$. Hence there exists a curve $\zeta:[0,1] \rightarrow \bar{\Omega}$ such that $\zeta(0)=x_{0}, \zeta(1) \in \partial \Omega$, and $\zeta^{*} \cap\left(N_{t_{0}} \cup I \cup J\right)=\varnothing$, where $\zeta^{*}$ is the image of $\zeta$. Since $x_{0} \in D\left(x_{j} ; \rho\right) \subseteq$ $\Omega_{t_{j}}$ for sufficiently large $j, \zeta^{*} \cap\left(N_{t_{j}} \cup I \cup J\right) \neq \varnothing$. Therefore there exist $y_{j} \in \zeta^{*}$ such that $v_{t_{j}}\left(y_{j}\right)=0$. Again by taking a subsequence we may assume that $y_{j} \rightarrow y_{0} \in \zeta^{*}$. Then $v_{t_{0}}\left(y_{0}\right)=\lim _{j \rightarrow \infty} v_{t_{j}}\left(y_{i}\right)=0$, hence $y_{0} \in N_{t_{0}} \cup I \cup J$ which is a contradiction since $\zeta^{*} \cap\left(N_{t_{0}} \cup I \cup J\right)=\varnothing$. Thus $A$ is closed. The proof for $B$ is similar. q.e.d.

Now we can finish the proof of Theorem 3.1. By Lemma 3.4, $A$ and $B$ are closed subsets of $\mathbb{R}$. By Lemma 3.2 we have $A \cap B=\varnothing$ and $A \cup B=\mathbb{R}$, and by Lemma 3.3 both $A$ and $B$ are nonempty. This is a contradiction since $\mathbb{R}$ is connected. Hence Theorem 3.1 is proved, and now Theorem 1.1 follows from Theorems 2.1 and 3.1.

4.

Proof of Lemma 2.1. (i) From the boundary regularity of elliptic differential equations it follows that $u$ is $C^{\infty}$ up to the boundary near $q$. Let $H=\left\{\left(x_{1}, x_{2}\right): x_{2}>0\right\}$ be the upper halfplane. Let $f: H \rightarrow \Omega$ be a conformal mapping of $H$ onto $\Omega$. By a theorem of Kellogg [6] $f$ extends $C^{\infty}$ to the boundary of $H$, and we may assume $f(0)=q$. Let $v=u \circ f$ in $\bar{H}$. Then there exists sufficiently small $\bar{r}_{0}>0$ such that $v$ is $C^{\infty}$ up to the boundary in $D\left(0 ; \bar{r}_{0}\right) \cap \bar{H}$ and $v=0$ in $D\left(0 ; \bar{r}_{0}\right) \cap \partial H$. By (2.1) we have $|\Delta v|=\mid \Delta u)\left.\circ f|| f^{\prime}\right|^{2} \leq C|v|$ in $D\left(0 ; \bar{r}_{0}\right) \cap \bar{H}$ for some $C$. Define $\tilde{v}$ on $D\left(0 ; \bar{r}_{0}\right)$ by

$$
\tilde{v}\left(x_{1}, x_{2}\right)= \begin{cases}v\left(x_{1}, x_{2}\right) & \text { if } x_{2} \geq 0 \\ -v\left(x_{1},-x_{2}\right) & \text { if } x_{2} \leq 0\end{cases}
$$

Then it is easy to check that $\partial \tilde{v} / \partial x_{1}, \partial \tilde{v} / \partial x_{2}, \partial^{2} \tilde{v} / \partial x_{1} \partial x_{2}$, and $\partial^{2} \tilde{v} / \partial x_{1}^{2}$ are continuous on $D\left(0 ; \bar{r}_{0}\right)$, and moreover $\partial^{2} \tilde{v} / \partial x_{1}^{2}=0$ in 
$D\left(0 ; \bar{r}_{0}\right) \cap \partial H$ since $v=0$ on $D\left(0 ; r \bar{r}_{0}\right) \cap \partial H$. From the inequality $|\Delta v| \leq C|v|$ in $D\left(0 ; \bar{r}_{0}\right) \cap \bar{H}$, it follows that $\partial^{2} \tilde{v} / \partial x_{2}^{2}$ is also continuous in $D\left(0 ; \bar{r}_{0}\right)$. Since $v$ is $C^{\infty}$ up to the boundary in $D\left(0 ; \bar{r}_{0}\right) \cap \bar{H}$, we conclude that $\tilde{v}$ is $C^{2,1}$ in $D\left(0 ; \bar{r}_{0}\right)$, and moreover $|\Delta \tilde{v}| \leq C|\tilde{v}|$ in $D\left(0 ; \bar{r}_{0}\right)$. Thus by Aronzajn's unique continuation theorem [1] $\tilde{v}$ does not vanish of infinite order in $L^{1}$-mean at 0 . Since $v$ is $C^{\infty}$ up to the boundary in $D\left(0 ; \bar{r}_{0}\right) \cap \bar{H} v$ does not vanish of infinite order at 0 . Hence $u$ does not vanish of infinite order at $q$.

(ii) Assume $q=0$. By (i) we may write

$$
u(x)=p_{m}(x)+O\left(|x|^{m+1}\right), \quad|x| \text { small }, x \in \Omega,
$$

where $p_{m} \neq 0$ is a homogeneous polynomial of degree $m$. Since $\Delta u+\lambda u=$ 0 in $\Omega$ we have

$$
\Delta p_{m}(x)=O\left(|x|^{m-1}\right) \quad \text { in } \Omega \text { for small } x .
$$

Since $\Delta p_{m}(x)$ is homogeneous of degree $m-2$ and the $x_{1}$-axis is tangent to $\partial \Omega$ at 0 , we conclude that $\Delta p_{m}(x)=0$ in $H=\left\{\left(x_{1}, x_{2}\right): x_{2}>0\right\}$, provided that the $x_{2}$-axis is in the direction of the inward normal to $\partial \Omega$ at 0 . Since $u=0$ on $D\left(0 ; r_{0}\right) \cap \partial \Omega$, by (4.2) we also have that $p_{m}(x)=0$ on $\partial H$, so that reflection $p_{m}$ is a harmonic polynomial vanishing on $\partial H$. Thus introducing polar coordinates, we have

$$
p_{m}(r, \theta)=c r^{m} \sin m \theta, \quad \text { where } c \neq 0 .
$$

For $r>0$ sufficiently small let $0 \leq \theta^{-}(r)<\theta^{+}(r) \leq \pi$ be the unique angles with $\left(r, \theta^{ \pm}(r)\right) \in \partial \Omega$. If $(r, \theta) \in \Omega \cap N$ and $r<r_{0}$, then

$$
u\left(r, \theta^{-}(r)\right)=u(r, \theta)=u\left(r, \theta^{+}(r)\right)=0 \quad \text { and } \quad \bar{\theta}<\theta<\theta^{-}(r) .
$$

Therefore there exist $\theta_{1}$ and $\theta_{2}, \theta^{-}(r)<\theta_{1}<\theta<\theta_{2}<\theta^{+}(r)$, such that $\partial u / \partial \theta\left(\left(r, \theta_{i}\right)\right)=0$ for $i=1,2$. By (4.2) and (4.4) we conclude that

$$
\left|\cos m \theta_{i}\right| \leq C_{1} r \quad \text { for some constant } C_{1} \text { and } i=1,2 .
$$

If $r<\left(2 C_{1}\right)^{-1}, c_{1}=\pi / 4 m>0$, and $c_{2}=\pi-\pi / 4 m<\pi$, then $\theta_{1}>c_{1}$ and $\theta_{2}<c_{2}$, and thus we have the following: If $(r, \theta) \in \Omega \cap N$ and $r<\left(2 C_{1}\right)^{-1}$, then $0<c_{1}<\theta<c_{2}<\pi$. Hence if $0 \in N$, then $N$ approaches 0 nontangentially with respect to $\partial \Omega$.

(iii) Clearly there is nothing to prove if $0 \notin N$. So we assume that $0 \in N$. Then by (ii) there exist $c_{1}, c_{2}, 0<c_{1}<c_{2}<\pi$, and $\delta>0$ such that if $(r, \theta)$ is in $\Omega \cap N$ and $0<r<\delta$, then $c_{1}<\theta<c_{1}$ and therefore 
$\sin \theta>c_{3}$, where $c_{3}=\min \left\{\sin \theta_{1}, \sin \theta_{2}\right\}>0$. Also from (4.2) and (4.4) it follows that

$$
\frac{\partial u}{\partial x_{1}}(r, \theta)=m c r^{m-1} \sin (m-1) \theta+O\left(r^{m}\right) .
$$

If $(r, \theta)$ is in $\Omega$ and $\partial u / \partial x_{1}(r, \theta)=u(r, \theta)=0$, then $|\sin m \theta| \leq$ $C_{2} r$ and $|\sin (m-1) \theta| \leq C_{2} r$ for some constant $C_{2}$. These imply that $|\sin \theta| \leq 2 C_{2} r$.

On the other hand, if $0<r<\delta$ and $u(r, \theta)=0$, then $\sin \theta>c_{3}$. Hence if $\varepsilon=\min \left\{\delta,\left(2 C_{2}\right)^{-1} c_{3}\right\}>0$, we have $|u|+\left|\partial u / \partial x_{1}\right|>0$ in $\Omega \cup\{x: 0<|x|<\varepsilon\}$.

\section{Acknowledgment}

The author wishes to thank Professors L. Carleson, S.-Y. Alice Chang, and S.-Y. Cheng for their interest in this work.

\section{Bibliography}

[1] N. Aronzajn, A unique continuation theorem for solutions of elliptic partial differential equations of second order, J. Math. Pures Appl. 36 (1957) 235-249.

[2] S.-Y. Cheng, Eigenfunctions and nodal sets, Comment. Math. Helv. 51 (1976) 43-55.

[3] R. Courant \& D. Hilbert, Methods of mathematical physics, Vol. 1, Interscience, New York, 1953.

[4] W. K. Hayman, Some bounds for principal frequency, Appl. Anal. 7 (1978) 247-254.

[5] D. Jerison, The first nodal line of a convex planar domain, Internat. Math. Res. Notices, No. 1, 1991; Duke Math. J. 62 (1991).

[6] D. Kellogg, Harmonic functions and Green's integral, Trans. Amer. Math. Soc. 13 (1912) 109-132.

[7] C.-S. Lin, On the second eigenfunctions of the Laplacian in $\mathbb{R}^{2}$, Comm. Math. Phys. 111 (1987) 161-166.

[8] L. E. Payne, Isoperimetric inequalities and their applications, SIAM Rev. 9 (1967) 453488.

[9] __, On two conjectures in the fixed membrane eigenvalue problem, Z. Angew. Math. Phys. 24 (1973) 720-729.

[10] S.-T. Yau, Problem section, seminar on differential geometry, Annals. of Math. Studies, No. 102, Princeton University Press, Princeton, NJ, 669-706.

University of CALIFornia, Los ANGeles 
